\title{
Programa de alfabetización musical en las comunidades yaquis de Pótam y Tórim
}

Cristian Salvador Islas Miranda Instituto Tecnológico de Sonora Departamento de Sociocultural cristian.islas@itson.edu.mx

\section{Resumen}

El objetivo del presente capítulo es describir los resultados de la implementación del programa alfabetización musical en las comunidades yaquis de Pótam y Tórim; proyecto financiado por el Programa de Apoyo a las Culturas Municipales y Comunitarias (PACMyC), convocatoria 2017; que se diseñó con el objetivo de capacitar en la lectura de la gramática musical a los músicos tradicionales adultos, a través de sesiones sabatinas de cuatros horas de duración en un lapso de seis meses. Para tal fin se seleccionaron los centros culturales de cada pueblo para impartir los cursos de manera gratuita. Se contó con el apoyo de los promotores culturales yaquis en la difusión y convocatoria de las actividades. Los contenidos de los cursos se adaptaron de acuerdo a los resultados de un diagnóstico, tomando en cuenta los intereses de los asistentes para su capacitación. Por último, se describe las metodologías educativas empleadas en el trabajo de campo y desarrollo del programa.

\section{Introducción}

La música es una de las expresiones artísticas más utilizadas alrededor del mundo tanto en el contexto rural como en el urbano, porque es a través de ella que se adquiere características propias de cada grupo, adaptada a su estructura simbólica, ya que es empleada en ceremonias y en distintos espacios colectivos que dan cuenta de las particularidades de los distintos grupos.
Las sociedades indígenas del noroeste de México, al igual que en otras partes del territorio nacional, han tenido la fortaleza y apego necesario para seguir conservando una parte importante, de lo que muy austeramente se podría reconocer como su patrimonio musical; en el caso de la comunidad yaqui, ubicada en el estado de Sonora, se ve reflejada en todas sus fiestas y ceremonias, por ejemplo en los diálogos melódicos del arpa y la guitarra que acompañan al pascola o las complejas células polirrítmicas en los instrumentos idiófonos que acompañan al venado.

Hoy en día, la formación musical (tradicional) de los habitantes de la comunidad yaqui está en manos de músicos de la tercera edad y no siempre pueden asegurar la continuidad de su legado, por lo que resulta evidente la necesidad de capacitar a las nuevas generaciones en la lectura de partituras con el objetivo de que realicen sus propios registros y creaciones musicales en partituras para que los futuros músicos las puedan interpretar a través de su lectura.

La pertinencia del programa alfabetización musical se basó, principalmente, en el hecho de que la música en la comunidad yaqui juega un papel muy importante en su ecología simbólica, no sólo como entretenimiento sino en sus celebraciones populares de índole religiosa (Varela, 1986), por lo que es concebida como objeto sagrado del culto y de carácter social (Tabla 1). Fuera de este marco se encuentran los cantos populares destinados al pasatiempo y goce de los mismos produ- 


\begin{tabular}{|c|c|c|c|l|}
\hline \multicolumn{5}{|c|}{ Breve Panorama acerca de la música, danza y personajes rituales entre los } \\
indígenas del noroeste.
\end{tabular}

Tabla 1: Música y danzas de la comunidad yaqui. Fuente: (Fragmento) Los pueblos indígenas del Estado de Sonora. Atlas etnográfico (Moctezuma, 2013, p. 346). Las palabras en cursiva fueron agregadas por Cristian S. Islas Miranda.

cidos por agrupaciones musicales locales, interpretando géneros musicales como la cumbia, el norteño, la banda, el mariachi, entre otras.

Los Centros de Cultura Indígena de Sonora fueron fundados a partir de 1983 con el objetivo de resguardar los instrumentos musicales tradicionales (arpas, guitarras, tambores e idiófonos utilizados en la danza del venado y pascola) y objetos ceremoniales. Hoy en día cuentan, además, con un acervo bibliográfico de consulta en diferentes áreas de conocimiento como historia, filosofía, literatura, música y teatro. El interior del recinto se utiliza para realizar talleres de lengua materna, música tradicional (violín y guitarra) y artes plásticas (dibujo) dirigidos a la comunidad en general, principalmente público infantil y juvenil. El exterior, una vez al año, alberga los encuentros de pascola y venado y el de música popular yaqui.

\section{Metodología}

Para la realización del presente trabajo se empleó el enfoque cualitativo a través del método de investigación-acción cuyas fases fueron basados en Bernal (2006):

Contacto con la comunidad.

Elaboración de un plan de acción.

Ejecución y evaluación del estudio.

Se incentivó el aprendizaje colectivo a través de diferentes técnicas pedagógicas en la búsqueda de que el conocimiento fuera significativo para que los asistentes se apropiaran de él. No se requirió nociones previas de teoría musical sólo de ejecución de algún instrumento, ya fuera de viento o cuerda.

Se solicitó autorización de la Dirección del Centro de Culturales Populares $e$ Indígenas de Cajeme para ingresa y dis- 
poner de los recintos culturales. Una vez aprobado, se trabajó del 02 de septiembre de 2017 al 25 de noviembre del 2017 en el Centro de Cultura Yaqui de Tórim Capitán "Manuel Molina León"; y del 02 de diciembre del 2017 al 24 de febrero del 2018 en el Centro de Cultura Yaqui de Pótam Capitán "Juan María Santeamea". En ambos espacios se efectuaron sesiones sabatinas, gratuitas, con un horario de las 10 a las 15 horas. El proyecto fue financiado por el Programa de Apoyo a las Culturas Municipales y Comunitarias (PACMyC) en la convocatoria 2017. Para la difusión de actividades, los promotores culturales de cada centro cultural ejercieron un rol muy importante, porque al tener identificados a algunos usuarios y a músicos tradicionales, los invitaron de manera personal.

Al final se contó con la participaron $9 \mathrm{mu}-$ jeres adolescentes y 17 hombres ( 8 niños, 7 jóvenes y 2 adultos). La convocatoria a las clases fue totalmente libre y de asistencia voluntaria. No se utilizaron medios electrónicos o redes sociales para difundir la publicidad, porque el acceso al internet es escaso así que se utilizaron medios tradicionales como las invitaciones publicadas en carteles, escritos a mano, que se colocaron en el cerco del mismo centro.

Para la recolección de información se aplicaron los siguientes instrumentos: 1 . Entrevista semiestructurada, la cual estaba constituida por cinco reactivos de respuesta abierta cuyas categorías giraron en torno a las actividades de los centros culturales, tipo de talleres que se ofrecen, sus horarios y cuál es la reacción de las persona, se aplicó solamente a los promotores culturales; 2. Bitácora donde se plasmó cada uno de los momentos de la sesión, reacciones de los usuarios así como comentarios y percepciones generales; 3 . Grabación de video y fotografía de los ejercicios realizados en el papel por los estudiantes.

\section{Procedimiento de la intervención pedagógica}

Los procedimientos de la implementación del programa alfabetización musical consistieron en:

Elaboración del programa de clase y actividades de los talleres con base en un diagnóstico.

Adquisición de material didáctico y papelería.

Taller de música en Tórim.

Taller de música en Pótam

Evaluación.

\section{Resultados}

Cuando se diseña un proyecto se visualiza un posible escenario y una respuesta de la comunidad con base en los objetivos específicos planteados, sin embargo se desconoce cómo será la reacción de la población a la que se intervendrá, a pesar de que se haya realizado un diagnóstico previo y detectado necesidades específicas. En el caso del programa alfabetización musical, se proyectó inicialmente para capacitar a los músicos tradicionales, ejecutantes de guitarra y arpa, pero estos no atendieron la convocatoria, fueron los niños y jóvenes quienes se acercaron de manera puntual a cada una de las sesiones, hasta que se convirtieron en asiduos estudiantes de los temas programados, como se menciona a continuación:

\section{Tórim}

Tórim es una comunidad indígena yaqui con más de 360 años de existencia. Fue fundado en 1617 cuando los yaquis aceptaron la presencia de misioneros jesuitas; quienes posteriormente organizaron a los miembros de la comunidad en ocho pueblos a lo largo del Río Yaqui. El nombre de Tórim proviene de la lengua cahita que significa: Rata de Campo. Especie muy común, conocida como neotoma, y abundante en ese lugar (Esquer, 2012). 
El primer contacto con el centro cultural fue a través de la promotora María del Rosario Hernández Urzúa, quien ha sido encargada del centro de cultura yaqui de Tórim Capitán "Manuel Molina León" desde hace más de 25 años. Es la coordinadora de las actividades culturales que se realizan en el centro como: talleres artísticos, encuentros, reunión de promotores, guía turística. Se llevó a cabo una entrevista semiestructurada para conocer los usos y costumbres de la comunidad e identificar las características de los talleres que se desarrollan, horarios y la respuesta de la población a las propuestas de formación artísticas. Cabe mencionar que desde un inicio mostró interés en que se realizara el taller de música y disposición para ayudar en los que se requiriera. Una vez establecidos los acuerdos de colaboración, se adquirió la papelería y material necesario para utilizarlo en las clases (Imagen 1).

En la primera sesión, se contó con la presencia de cinco asistentes, de 8 a 21 años, quienes respondieron a la invitación de la promotora cultural. No contaban con conocimientos previos de teoría musical, a diferencia de lo que se esperaba, ya que se trataba de músicos tradicionales o populares para su capacitación en la lectura de música.

Para conocer a los educandos, se realizó una actividad de integración grupal que consistió en presentarse de manera libre diciendo su nombre, edad, escolaridad, si tocaban algún instrumento, intereses musicales y expectativas del curso. Entre las respuestas más repetidas se mencionó que: 1. Todos tenían un familiar (padre, tío, primo) que tocaba algún instrumento, en su mayoría guitarra o violín; 2 . Nunca habían llevado clases de música; 3. Les interesaba aprender a tocar la guitarra popular; 4. Conocían los acordes musicales, pero no sus nombres; 5. Les llamaba la atención observar a los músicos en las fiestas. 6. Tiene especial interés en la música banda y norteña.

Con base en la información proporcionada, se realizó un ajuste en los contenidos del programa priorizando la enseñanza de la ejecución de la guitarra popular con el sistema anglosajón para que los estudiantes pudieran escribir y ejecutar los acordes que acompañan las melodías de cualquier género musical.

A partir de la segunda sesión, hubo un incremento de la asistencia, oscilando entre 13 y 15 estudiantes, con el mismo nivel de conocimiento en el área musical e interés por aprender a ejecutar la guitarra. Para atender al número de interesados, se utilizaron las guitarras acústicas facilitadas por el centro cultural.

Contenido

\begin{tabular}{l}
\hline Tema 1. Ejercicios de digitación: mano izquierda. \\
Objetivo: Identificar las cuerdas y trastes de la guitarra mediante ejercicios de digitación \\
utilizando los dedos 1,2, 3 y 4 de la mano izquierda. \\
\hline Tema 2. Mis primeros acordes: EM-Am. \\
Objetivo: Ejercitar la memoria muscular mediante la ejecución de acordes sencillos con \\
acompañamientos ritmico básicos. \\
\hline Tema 3. Ejercicio de coordinación mano derecha con mano izquierda. \\
Objetivo: Distinguir la diferencia entre arpegio y rasgueo de la mano derecha al \\
acompañar acordes básicos con la mano izquierda. \\
\hline Tema 4. Mis primeros acordes: EM-Am-GM-Em-DM \\
Objetivo: Distinguir la posición de los acordes mayores y menores en los trastes de la \\
guitarra mediante ejercicios de acompañamiento rítmico básico. \\
\hline Tema 5. Nombre de los acordes y su escritura. \\
Objetivo: Interpretar los acordes de la guitarra utilizando la nomenclatura inglesa. \\
\hline Tema 6. Círculo de GM y DM. \\
Objetivo: Distinguir los acordes que conforman los círculos de Sol mayor y de Re mayor \\
utilizando ejercicios de acompañamiento rítmico básicos. \\
\hline Tema 7. Los principales círculos empleados en la música popular. \\
Objetivo: Distinguir los círculos de CM, DM, EM, FM, GM, AM y BM. Utilizando \\
ejercicios de acompañamiento rítmico básico. \\
\hline Tema 8. Cómo interpretar una canción escrita. \\
Objetivo: Descifrar el acompañamiento musical que se utiliza en las canciones \\
populares con los ejercicios de acompañamiento correspondiente al género musical. \\
\hline
\end{tabular}




\section{Observaciones}

En el desarrollo de las clases se detectó interés en los temas abordados. Antes iniciar la sesión ensayaban los ejercicios de una clase anterior y entre ellos se apoyaban dándose consejos de cómo realizar las diferentes posiciones de los acordes, también solían cargar con alguna piedra para colocarlas como pisa papel y no batallar con el aire que movía sus hojas.

Como producto final los estudiantes presentaron un programa musical en el jardín del centro cultural, con una duración de veinte minutos, que consistió en la interpretación de tres piezas populares y una selección de los primeros ejercicios realizados en clase: combinación de acordes y círculos armónicos. Se contó con la asistencia de familiares.

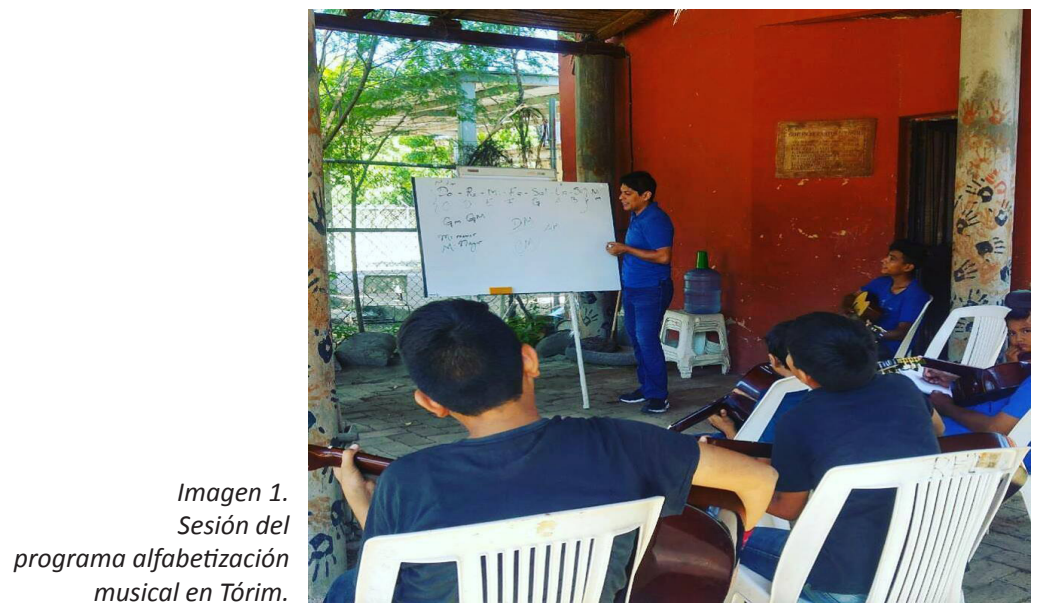

\section{Pótam}

Pótam es un pueblo que está situado en el municipio de Guaymas. El nombre de Pótam proviene de la lengua cahita que significa: Topos.

El primer contacto con el centro cultural fue a través del promotor cultural y profesor Ismael Castillo Rendón, quien es el encargado del Centro de Cultura Yaqui de Pótam Capitán "Juan María Santeamea" desde hace más de 20 años. Además de sus actividades de promoción cultural, es músico tradicional y profesor de guitarra, violín, arpa, vihuela y guitarrón. Es fundador del primer mariachi femenil “"'Jiak
Usim Jiawai" (Imagen 2). A diferencia de la experiencia de Tórim, el profesor Ismael Castillo solicitó que se capacitará a las integrantes del mariachi a leer partituras porque, en sus palabras: "a mí me enseñaron unas monjas, pero nunca le entendî", por lo tanto conocía las ventajas que conlleva aprender a leer música.

Al igual que en Tórim, también se le realizó una entrevista diagnóstica para conocer los conocimientos previos de los aspirantes e identificar las características de los talleres que se desarrollan en el centro, horarios y cómo es la respuesta de la población a las propuestas de formación artísticas. A pesar de que se lanzó la convocatoria para toda la comunidad y se publicó en el exterior del recinto, sólo asistieron las integrantes del mariachi.

En la primera sesión de clase se contó con la presencia de las ocho integrantes del mariachi, quienes acudieron porque el profesor les solicitó que asistieran de manera obligatoria. No contaban con conocimientos previos de teoría musical, pero sí de ejecución instrumental, por lo que tenían facilidad de interpretar melodías populares en los instrumentos de cuerda: violín, charango, guitarra.

Para conocer a las asistentes, se realizó una actividad de integración grupal que consistió en presentarse de manera libre diciendo su nombre, edad, escolaridad, cuánto tiempo llevaban tocando el instrumento en el ensamble, intereses musicales y expectativas del curso. Entre las respuestas más repetidas se mencionó que: 1 . Todas tenían más del año como integrantes del mariachi; 2. Nunca habían llevado clases de teoría musical, lo que sabían era lo que les había enseñado el profesor Ismael Castillo; 3. Les interesaba aprender a leer notas musicales; 4. Conocían los acordes musicales, pero no cómo se escribían; 5. Tenían experiencia tocando en las fiestas tradicionales yaquis, a pesar de que en un inicio no fue bien visto por los hombres. 6. Tenían especial interés en la música banda, pop comercial y norteña. 
Con base en la información proporcionada, se realizó nuevamente ajustes en los contenidos del programa priorizando la enseñanza de la lectura musical para que pudieran ejecutar melodías desde la partitura con su respectivo instrumento.

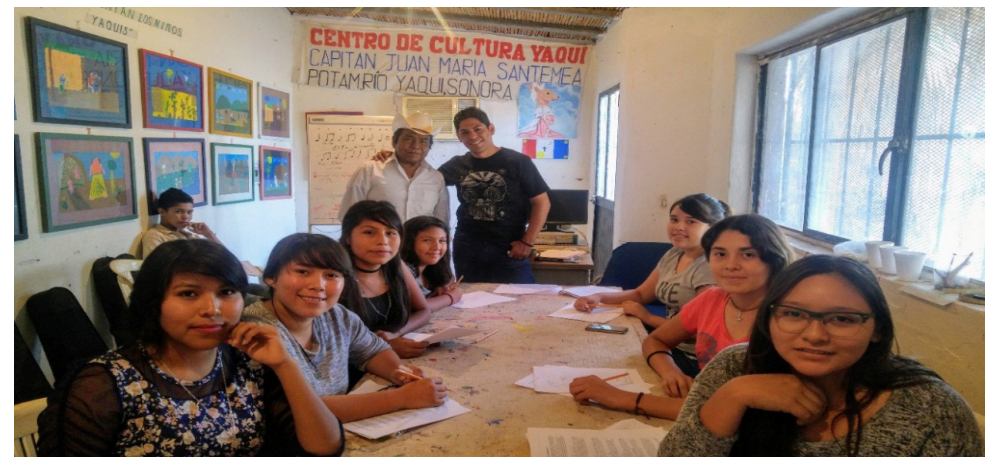

Imagen 2.

Con el profesor Ismael Castillo Rendón y las Integrantes del mariachi femenil: "Jiak Usim Jiawai" de Pótam.

Contenido

\begin{tabular}{|l|}
\hline Tema 1. Ejercicios de digitación: mano izquierda. \\
Objetivo: Identificar las cuerdas y trastes de la guitarra mediante ejercicios de digitación \\
utilizando los dedos 1, 2, 3 y 4 de la mano izquierda. \\
\hline Tema 2. Mis primeros acordes: EM-Am. \\
Objetivo: Ejercitar la memoria muscular mediante la ejecución de acordes sencillos con \\
acompañamientos ritmico básicos. \\
\hline Tema 3. Ejercicio de coordinación mano derecha con mano izquierda. \\
Objetivo: Distinguir la diferencia entre arpegio y rasgueo de la mano derecha al \\
acompañar acordes básicos con la mano izquierda. \\
\hline Tema 4. Mis primeros acordes: EM-Am-GM-Em-DM \\
Objetivo: Distinguir la posición de los acordes mayores y menores en los trastes de la \\
guitarra mediante ejercicios de acompañamiento ritmico básico. \\
\hline Tema 5. Nombre de los acordes y su escritura. \\
Objetivo: Interpretar los acordes de la guitarra utilizando la nomenclatura inglesa. \\
\hline Tema 6. Círculo de GM y DM. \\
Objetivo: Distinguir los acordes que conforman los círculos de Sol mayor y de Re mayor \\
utilizando ejercicios de acompañamiento rítmico básicos. \\
\hline Tema 7. Los principales círculos empleados en la música popular. \\
Objetivo: Distinguir los círculos de CM, DM, EM, FM, GM, AM y BM. Utilizando \\
ejercicios de acompañamiento rítmico básico. \\
\hline Tema 8. Cómo interpretar una canción escrita. \\
Objetivo: Descifrar el acompañamiento musical que se utiliza en las canciones \\
populares con los ejercicios de acompañamiento correspondiente al género musical. \\
\hline
\end{tabular}

\section{Observaciones}

La experiencia en Pótam fue lo más apegado al objetivo inicial del proyecto alfabetización musical, el hecho de que las integrantes tuvieran conocimientos en la ejecución de un instrumento musical favoreció que las sesiones y los contenidos fueran más significativos. Por lo tanto se agilizó el trabajo con los contenidos programados y permitió que las estudiantes realizaran más ejercicios que los proyectos.

Sin lugar a dudas el apoyo del profesor y director del mariachi, Ismael Castillo Rendón, fue clave porque siempre estuvo monitoreando la asistencia y conducta de las estudiantes.

Al final presentaron un programa de quince minutos, compuesto por piezas populares mexicanas y obras clásicas occidentales con una extensión de doce compases. Todas las partituras fueron descifradas sin apoyo del docente.

\section{Conclusiones}

A manera de conclusión, se considera muy importante seguir fomentando la formación musical en los jóvenes de la comunidad yaqui, ya que en ellos radica el compromiso y disposición de aprender teoría que les servirá para elevar la 
calidad de su música e implementar nuevas técnicas de registro a través de los códigos musicales. Solo eso podrá derivar en un verdadero rescate, preservación y difusión de su cultura musical y cualquier otra; ya que dichos vestigios siempre han de encontrarse en los que logra heredarse entre generaciones.

Falta mucho por hacer, sin embargo en esta primera fase de implementación del proyecto alfabetización musical, se cumplió el objetivo ya que se asentaron las bases teóricas que permitirán al educando interpretar diferentes géneros musicales, pero se recomienda avanzar en una segunda o tercera aplicación del proyecto para que el nivel de dominio se incremente y el músico sea totalmente autónomo en el manejo de la gramática musical. El pilotaje del presente estudio servirá para afinar las estrategias de difusión o reclu- tamiento en futuros proyectos de capacitación musical en las comunidades indígenas.

Es inherente a lo anterior instar a los organismos interesados y comprometidos en el apoyo de los antepasados en todas las regiones del mundo, a continuar solventando proyectos comunitarios de diversa índole, para que la vasta riqueza que aún prevalece en las comunidades indígenas quede reconocida y continúe pasando por generaciones; ya tenemos demasiadas experiencias de solo conocer a las culturas desaparecidas por hallazgos y vestigios mínimos encontrados perdidos en la historia, no puede permitirse que eso siga ocurriendo. Es responsabilidad del gobierno sí, pero también es responsabilidad de las universidades y otros organismos que con sus diferentes grupos colegiados pueden aportar: haga cada quien lo suyo.

\section{Bibliografía}

Bernal, C. (2006). Metodología de la investigación. México: Pearson.

Castro, T. (Coord.), (2011). Etnias de Sonora. México: Mora-Cantúa Editores

Cruz, G. (2015). La escritura autobiográfica como recurso de reflexión crítica. Recuperado de: $\underline{\text { http://ucsj.redalyc.org/articulo.oa?id=13239889007 }}$

Esquer, R. (2012). Acciones de gobierno y su impacto en el índice de desarrollo humano de la comunidad de Tórim, Guaymas, Sonora 1997-1999. Recuperado de: http://www.eumed.net/tesis-doctorales/2013/rer/rer.pdf

ITESM (2017). Los Yaquis. Consultado en http://www.mty.itesm.mx/dhcs/deptos/co/ co95-832/Proy 2000 S2/CulturasDesierto/Culdes/yaquis.html

Moctezuma J. (2013). Los pueblos indígenas del Noroeste. Atlas etnográfico. Sonora, México:

Instituto Sonorense de Cultura.

Patrón, G. (2016). El progreso espiritual en Sonora. Expediciones, misiones, provincias y danzas yaquis. México: Asociación para las Bellas Artes, A.C., H. Ayuntamiento de Cajeme, Secretaría de Educación y Cultura del Gobierno de Sonora

Varela, L. (1986). La música en la vida de los Yaquis. Hermosillo, Sonora: Gobierno del Estado de Sonora. 